\title{
THE EFFECT OF MORPHINE ON THE RESPIRATION IN PNEUMONIA
}

By JOHN STAIGE DAVIS, JR.

(From the Hospital of The Rockefeller Institute for Medical Research, New York)

(Received for publication July 24, 1928)

\section{INTRODUCTION}

Opinion, as to the advisability of administering opiates to patients suffering from respiratory disorders, especially pneumonia, is divided. For the most part, the writers of the older text books of medicine were opposed to the use of morphine; however, in certain cases, they believed that codeine was justifiable. The modern writers, particularly Cecile (1) and Cole (2), are of the opinion that the opiates are very necessary and important drugs in the treatment of pneumonia. Cushny (3) states that severe pain indicates opium even when the disease itself is one which in ordinary circumstances would contraindicate it. Codeine is insufficient in severe pain. Hewlett (4) believes that codeine is the drug of choice for treatment of pain and cough in pneumonia. He agrees with Cushny that in cases where the respiration is barely sufficient to aerate the blood, or where profuse expectoration is present, morphine is dangerous, because of its depressant action on the respiratory center, the tendency to abdominal distention, and to the relaxation of the bronchial musculature and thus the danger of edema of the lung is increased.

Macht (5) has done experimental work on normal rabbits and puppies to determine the volume of air respired after morphine. He showed that when small doses of morphine are given the volume of air respired is increased and, what is more important, the alveolar ventilation is also increased, even though the rate be slowed. When, on the other hand, a full dose of morphine is given ( 1 to $5 \mathrm{mgm}$. per kilogram) the rate is markedly slowed, the total volume of air respired is diminished, and the alveolar ventilation is also diminished. The dead space is slightly increased, pointing to a bronchial dilatation. 


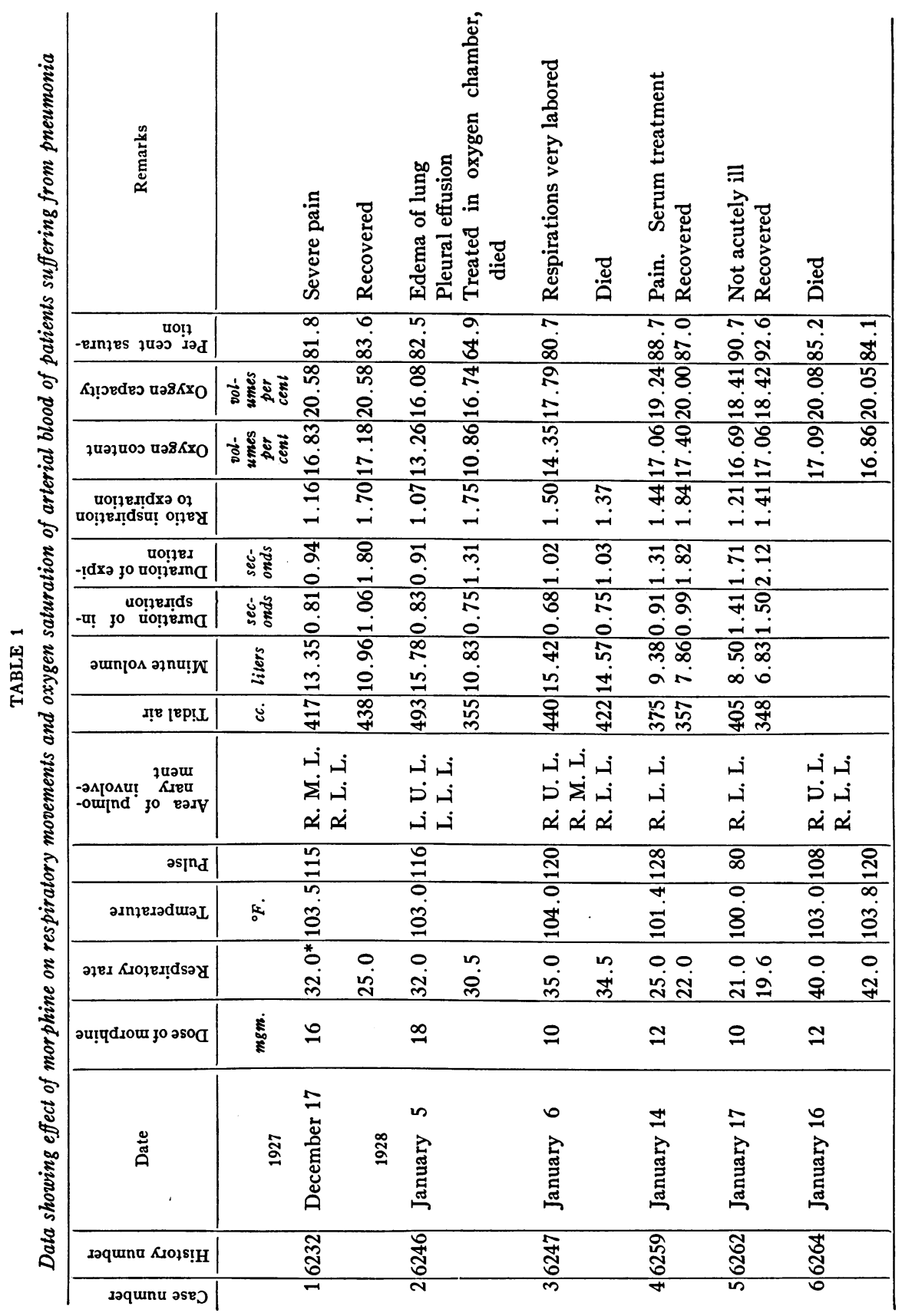




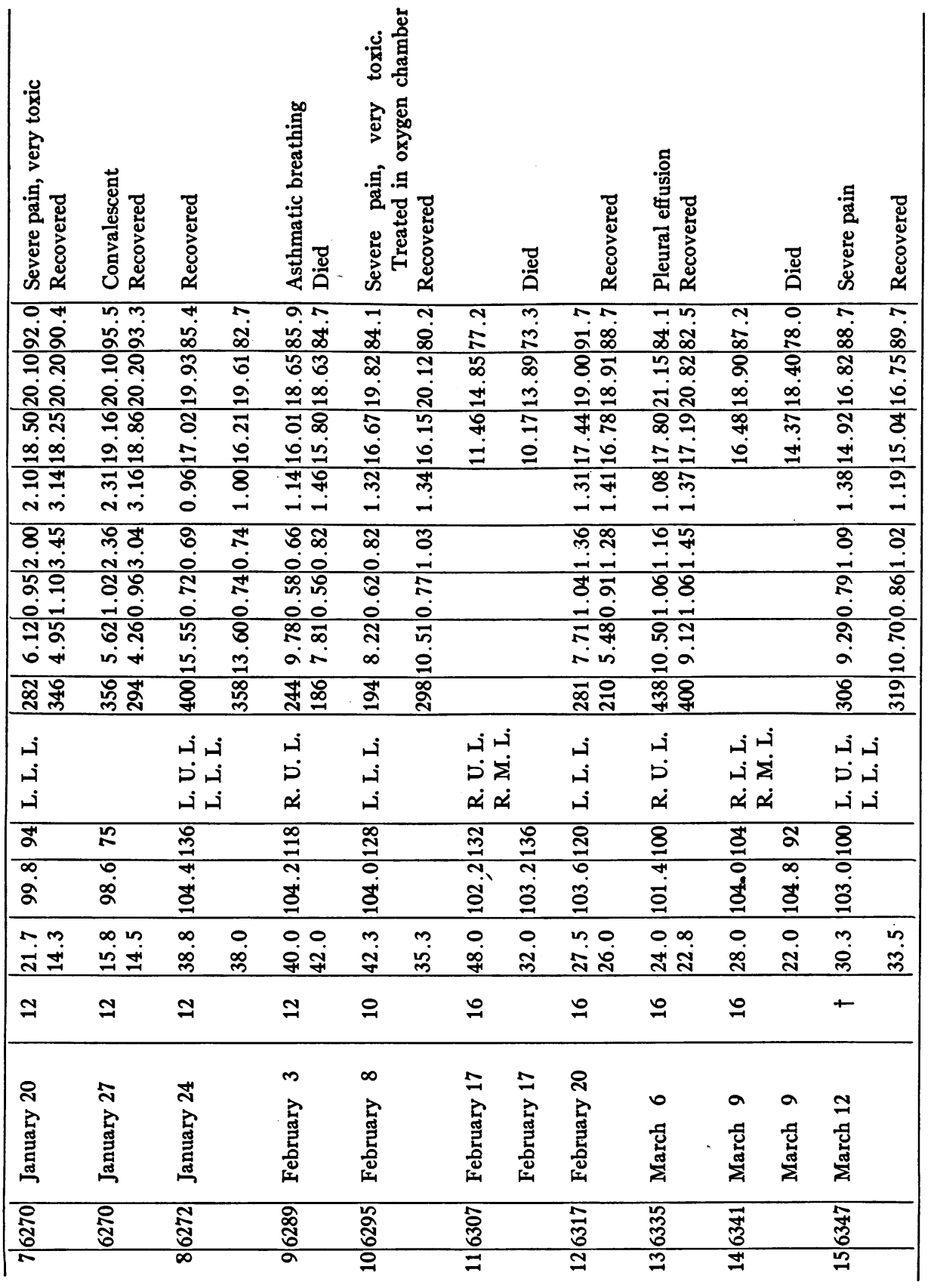




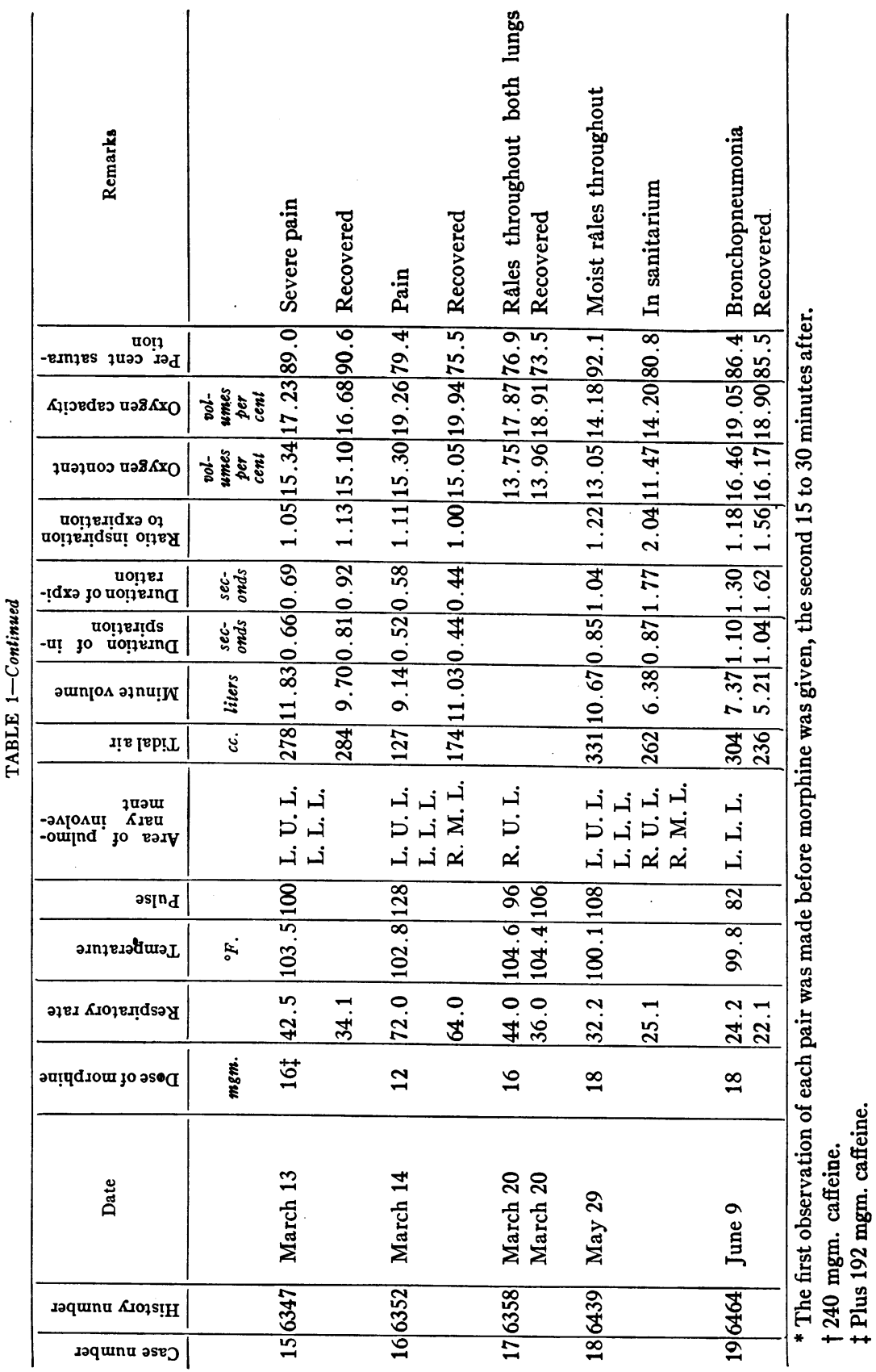


Codeine has a somewhat similar effect, with less influence on the total volume output.

Several investigators have carried out experiments showing the effect of morphine on the respiratory movements and gaseous metabolism of normal human beings. Higgins and Means (6) concluded from their experiments on three subjects that morphine in therapeutic doses acted as a respiratory depressant, sometimes by acting directly on the center and sometimes by bronchoconstriction, and in one instance by both. The total gaseous metabolism showed as a rule no change in oxygen consumption (or a very slight diminution) and a marked drop in $\mathrm{CO}_{2}$ elimination. The respiration rate usually showed a small increase.

The object of these experiments was to determine the effect of morphine on the respiratory movements and the oxygen saturation of the arterial blood of pneumonia patients: From these observations it was hoped that conclusions based on physiological evidence could be drawn concerning the value of morphine in the treatment of pneumonia.

\section{METHODS AND MATERIAL}

The observations have been made on human beings, the greater number of whom were acutely ill with lobar pneumonia. One-hundred and seventy-three observations have been made on 33 different cases of pneumonia. On these 43 arterial punctures were done. Fifteen patients were studied in a body plethysmograph recently described by us (7). They were observed in the plethysmograph from 50 minutes to $1 \frac{1}{2}$ hours An arterial puncture was done before and after each respiratory tracing was made. Morphine was given subcutaneously while the patient was in the plethysmograph and a second tracing was made from 15 to 30 minutes after the administration of the drug. The dose of morphine varied from 10 to $18 \mathrm{mgm}$. One patient was given caffeine in addition to morphine.

The oxygen analyses of the arterial blood were done by the method of Van Slyke and Neill (8). All estimations were made in duplicate.

\section{EXPERIMENTAL}

In order to simplify the interpretation of the experimental data, we have divided the observations into two parts: (I) The effect of 
morphine on the respiratory movements, and (II) the effect of morphine on the oxygen saturation of the arterial blood. The relationship of these two has been considered elsewhere (9). The data are tabulated in table 1.

I. The respiratory movements of 15 patients were studied in the plethysmograph before and after morphine administration.

1. Rate. In all but one case there was a drop in rate. The average drop in 14 cases was 4.1 to the minute. The greatest fall was from 42.5 to 34.1. In one patient (number 6289) the rate increased from 40 to 42 per minute. His tidal air, however, fell from 244 to $186 \mathrm{cc}$, and his minute volume from 9.78 to 7.81 liters. His breathing indicated edema of the lungs and at the time of observation he was rapidly growing worse. His arterial saturation dropped from 85.9 to 84.7 per cent after morphine, a change of little, if any, significance.

2. Tidal air. In 10 cases there was a drop in tidal air, the greatest drop being $138 \mathrm{cc}$. This occurred in patient number 6246, whose arterial saturation diminished 21.3 per cent. The average drop in tidal air was $57.7 \mathrm{cc}$. In the remaining 5 cases the tidal air increased, the greatest increase being $102 \mathrm{cc}$. In these 5 cases, pleuritic pain was a prominent symptom, and relief of pain by morphine probably allowed deeper breathing with more comfort. In one of the 5 cases $192 \mathrm{mgm}$. of caffeine were given with the morphine. These wll be considered later. In no one of these 5 cases was the increase in arterial saturation as great as 4 per cent.

3. Minute volume. In 13 cases the minute volume was diminished after morphine. The greatest drop in minute volume was 4.95 liters and the smallest was 0.85 liter, the average being 2.20 liters.

Two patients (numbers 6295 and 6352) showed an increase in minute volume. In both of these the breathing was extremely shallow and the rate correspondingly rapid. In the one case the rate was 42.3 and the tidal air was $196 \mathrm{cc}$., while in the other the rate was 72 and the tidal air was $127 \mathrm{cc}$. In the first patient the minute volume increased from 8.22 to 10.51 liters, and in the second it increased from 9.14 to 11.03 liters. Both patients were very ill. The first had two lobes involved, and the second had three. In neither were there any signs of edema of the lungs. In the second patient there seems to have been a psychic element in the excessively rapid respirations, 
since the rate increased at once when he was under observation, even though he was unaware of the fact that his respirations were an object of interest. In both these patients pleuritic pain was severe. The increase in minute volume following morphine administration is probably related to the relief of pain.

In 13 cases the ratio of expiration to inspiration increased, while in the other 2 cases there was a very slight drop in the ratio. The only significance to be attached to this is that the slowing of the respiratory rate produced by morphine is accomplished by prolonging the expiratory phase with little change in the inspiratory phase. What the functional interpretation of this is is not clear.

Twenty-seven patients on the ward were observed after receiving morphine. The observations are shown in more detail in table 2.

1. Rate. In all but one case the rate was slowed, the average drop being 4 and the greatest drop 14 to the minute. We have divided these 27 cases into 3 groups: (a) those who recovered and who never appeared to be in any great danger of death; $(b)$ those who recovered, but concerning whom a grave prognosis was given; $(c)$ those who died.

In the first group there are 12 cases, in the second 7, and in the third 8. The total dose of morphine and the time during which it was administered varied considerably, because this, of course, depended on the individual needs of each patient. It is difficult, therefore, to make an exact comparison of one group with the other. The differences in results, however, are sufficiently striking that certain general conclusions can be drawn. In group $(a)$ the average drop in rate after the total dose of morphine had been given was 2.0 , while in group (b) the drop was 7.3, and in group (c) it was 3.4 (table 2). It does not seem that the morphine had a much more depressant effect on the patients in group $(c)$ than it did on those in group $(a)$. If, however, one takes into consideration the fact that usually the respiratory rate gradually increases as a pneumonia patient approaches death, a drop in rate of 3.5 to the minute may be significant. Moreover, all of the facts are not shown by the figures, for in 2 cases (numbers 6307 and 6315) shortly before death the rate fell in the one to 10 and in the other to 18 , which in the first instance represented a drop of 32 to the minute and in the second a drop of 20 . This occurred more than 2 hours after morphine was given, so these figures are not in- 


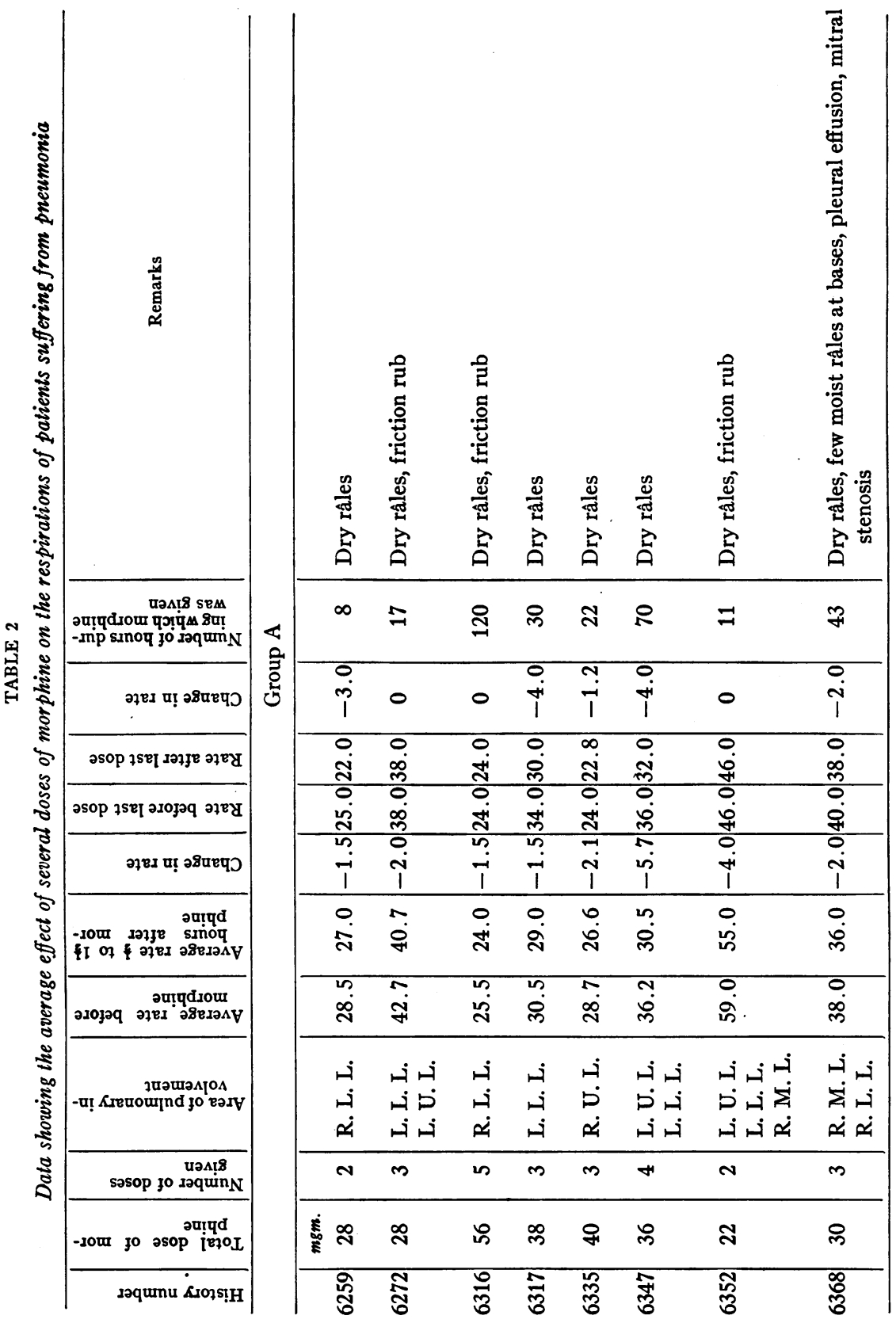


JOHN STAIGE DAVIS, JR.
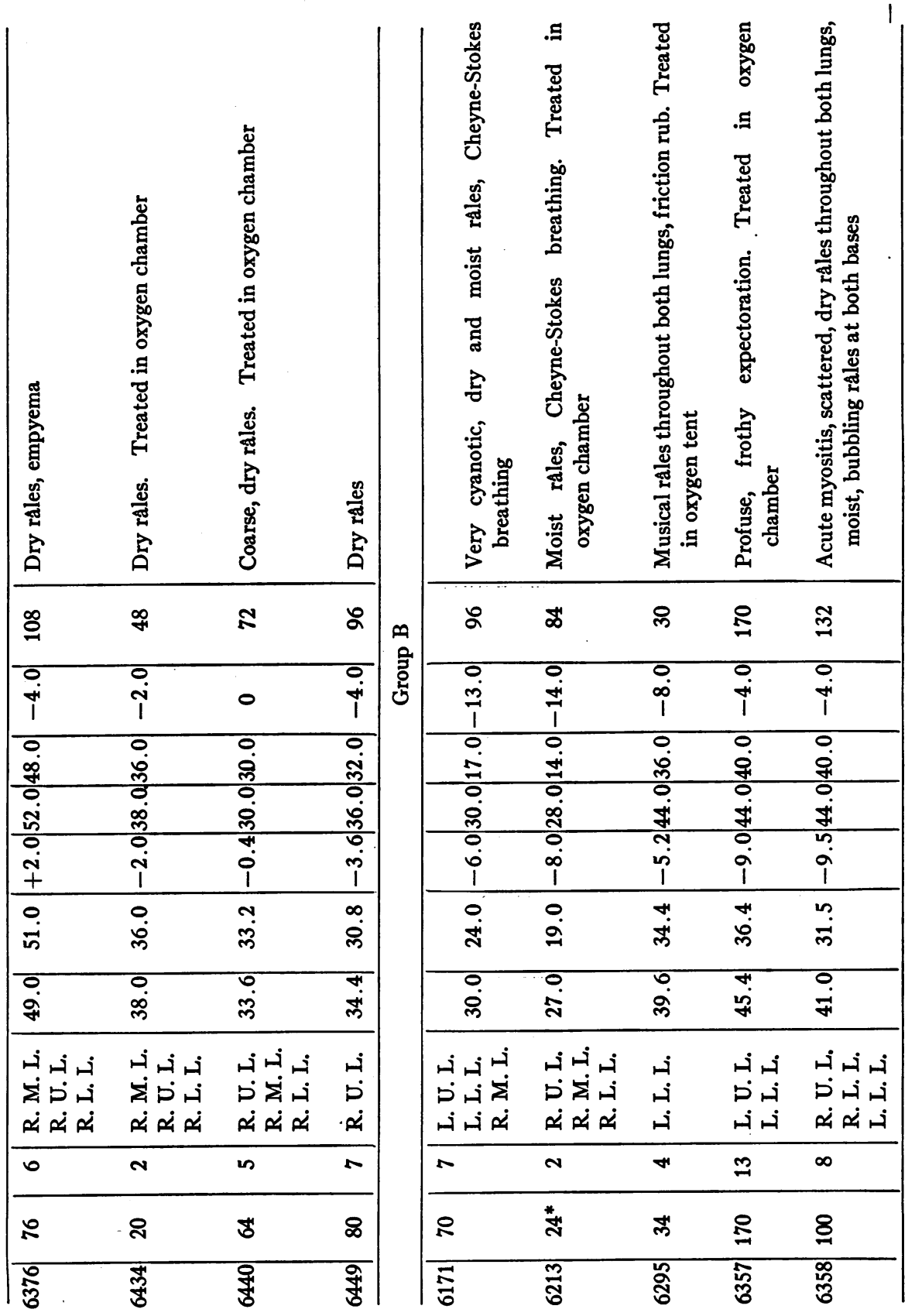

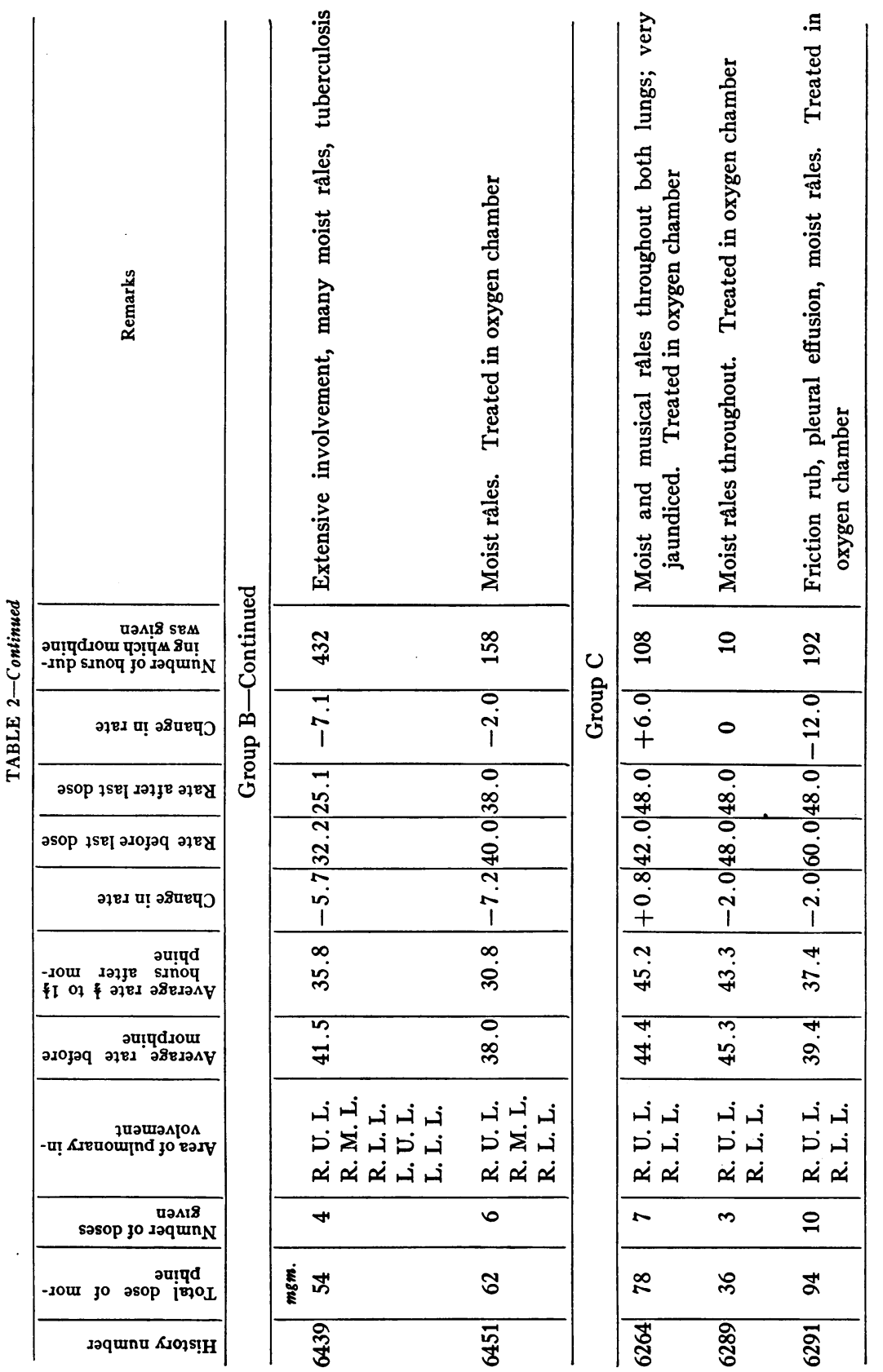


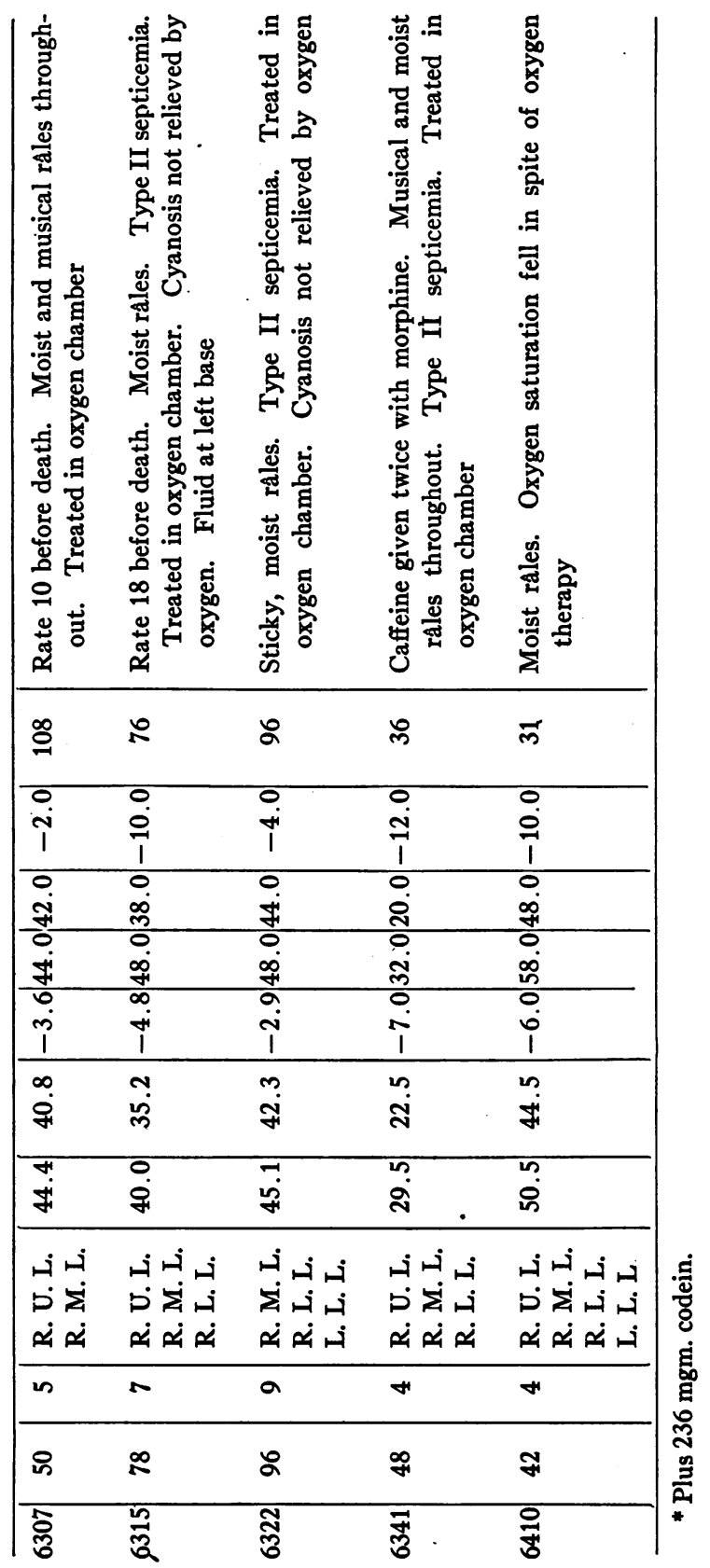


cluded in the table. We believe that this delayed slowing of the rate is in part at least due to the cumulative action of morphine.

We have also studied the effect of caffeine on the respiratory movements and arterial saturation of one patient (number 7347). He was observed on 2 successive days while acutely ill. On the first day he was given $240 \mathrm{mgm}$. of caffeine sodium benzoate, and on the second day he was given $192 \mathrm{mgm}$. of caffeine and $12 \mathrm{mgm}$. of morphine. After caffeine alone, his rate, tidal air; minute volume, and per cent saturation increased, while the morphine and caffeine together resulted in a reduction in rate, but a slight increase in tidal air and per cent saturation. The minute volume on this occasion was smaller after the drug was given than before. None of these changes was of sufficient magnitude to attach much importance to them, but at least they are different from those observed after morphine alone. That codeine in moderate amounts may have a detrimental effect on the respiratory center is suggested by case number 6213 , whose respiratory rate dropped to 14 and whose arterial saturation dropped to 40 per cent after he had had $24 \mathrm{mgm}$. of morphinein addition to $236 \mathrm{mgm}$. of codeine, which he had received during a period of $3 \frac{1}{2}$ days. His breathing was of the Cheyne-Stokes type, his lungs were filling with exudate, he rapidly passed into coma, and, had he not been immediately put into the oxygen chamber, he would in all probability have died. The next morning, while breathing a 40 per cent oxgen mixture, his arterial saturation was 90.3 per cent and his respiratory rate was 18.

II. Effect of morphine on the oxygen saturation of the arterial blood. In 16 patients out of 20 on whom oxygen analyses of the arterial blood were done before and after morphine, there was a drop in the arterial saturation. The greatest drop was 21.3 per cent and the average drop was 5 per cent (table 1 ). Of the remaining 4 cases, 3 showed a slight rise in $\mathrm{O}_{2}$ saturation after morphine. In one no analysis was made. One of these 3 cases (number 6232) had severe pain, which was relieved by morphine. His tidal air increased from 412 to $438 \mathrm{cc}$., though his minute volume dropped from 13.35 to 10.96 liters. Another, case number 6262, was not acutely ill, and the third (case number 6347) was given caffeine with the morphine. . Moreover, in his case pain was relieved by morphine and his tidal air increased. 
In only 3 cases was the change in oxygen saturation great enough to be of significance. (1) In patient number 6246 , the saturation fell from 82.5 to 64.9 per cent. This was in a man of 63 years, who had been sick for 3 days. He appeared cyanotic and dyspneic, signs of consolidation extended from the left apex to the base. At the left

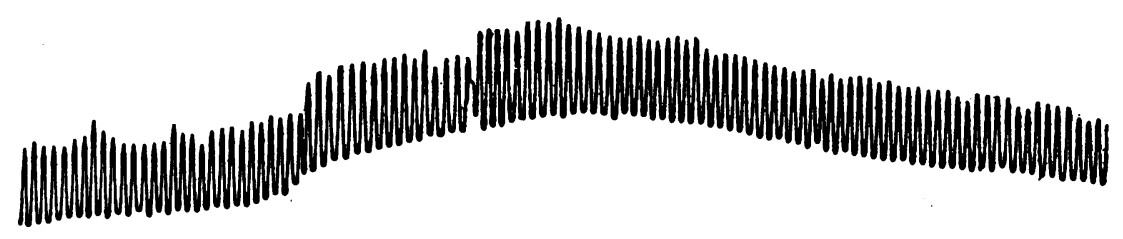

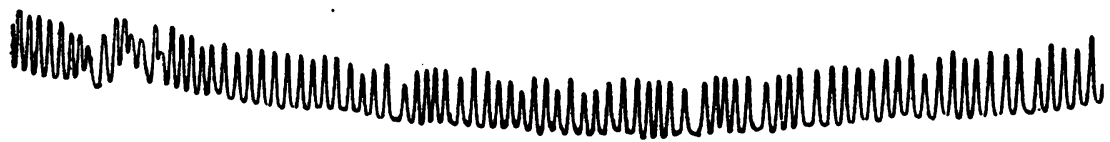

Fig. 1. (a) Plethysmographic Tracing from Case Number 6439, Made Before the Administration of Morphine; (b) Plethysmographic Tracing from Same Patient, Made 25 Minutes after 16 mgm. of Morphine Had BeEN GIVEN

The upper line was drawn by the work-adder signal lever. Interval between signal lever equals 12.34 liters. The lower line represents time in 2 second intervals.

base were signs of fluid. Moist râles were present in both lungs. His blood culture was positive for Pneumococcus Type III. (2) In case number 6341, the saturation fell from 87.2 to 78.7. This was in an obese woman of 50 years, who had had a severe chill 3 days previously. The right middle and lower lobes were involved. Throughout both 
lungs breath sounds were bronchovesicular and numerous rhonchi were heard. She appeared cyanotic. Blood culture was positive for Type II pneumococus. She was transferred to the oxygen chamber on the day the morphine test was made. The next day her arterial saturation was 93.2 per cent. Five days later she died. (3) In patient number 6439 the saturation fell from 92.1 to 80.8 per cent. He had been acutely ill for 12 days. Tubercle bacilli had been demonstarted in his sputum. His temperature fluctuated from $101^{\circ}$ to $104^{\circ} \mathrm{F}$. The only uninvolved part of his lungs was the lower right lobe, which was clear when the arterial punctures were done. Moist râles were present throughout both lungs. Figure $1, a$ and $b$, shows parts of his respiratory tracing before and after morphine administration. These 3 patients in whom morphine administration resulted in a definite and perhaps serious unsaturation of the arterial blood were all extremely ill. Moreover, they showed not only extensive lung involvement, but the presence of diffuse, moist râles. It is to be noted that in none was anoxemia severe before morphine. A fourth case already referred to is not included here, since no arterial blood analysis was made before morphine. After morphine injection, however, his saturation was only 40 per cent.

\section{DISCUSSION}

In most cases of pneumonia the effect of morphine on the respiratory movements and on the arterial oxygen saturation is slight. Certainly the depression of respiration which follows morphine administration is ordinarily not sufficient to contraindicate its use. The benefits which may accrue to the patient in the direction of relief from pain, reduction of metabolism, and sleep, undoubtedly outweigh the possible ill effects of a slight reduction in pulmonary ventilation and increase of anoxemia.

Occasionally, however, morphine may so diminish pulmonary ventilation as to result in serious oxygen want. This is liable to occur in patients in whom the pulmonary involvement is extensive and is accompanied by diffuse moisture, and in patients who are already suffering from severe oxygen want. Because of the possibility of this type of reaction to it, morphine must always be used with caution and is best combined with oxygen therapy. 


\section{SUMMARY AND CONCLUSIONS}

1. The respiratory rate, tidal air, and minute volume of pulmonary ventilation has been measured in 15 cases of pneumonia before and after administration of morphine.

2. In most instances a reduction in respiratory rate, tidal air, and minute volume follows morphine administration.

3. Accompanying the reduction in pulmonary ventilation there is, as a rule, a diminution in the $\mathrm{O}_{2}$ content of the arterial blood without a corresponding change in the $\mathrm{O}_{2}$ capacity. There occurs, therefore, a decrease in the per cent saturation of the arterial blood.

4. This change may be only a small one, but is usual. In only 3 cases out of 19 was it not observed. The average drop was 5 per cent. In one patient it was as great as 21.3 per cent.

5. When there is much pleuritic pain, the relief brought by morphine may allow the pulmonary ventilation to increase and thus raise slightly the per cent saturation of the arterial blood.

6. In the presence of an extensive pulmonary involvement with diffuse, moist râles morphine administration may result in a dangerous degree of anoxemia.

I wish to express my grateful appreciation to Dr. Carl A. L. Binger for his assistance and advice in this work.

\section{BIBLIOGRAPHY}

1. Cecil, R. L., Text Book of Medicine, Saunders \& Co., Philadelphia, 1927, 36.

2. Cole, Rufus, Nelson's System of Medicine, New York, 1920, i, 258. Acute Lobar Pneumonia.

3. Cushny, A. R., A Text-book of Pharmacology and Therapeutics, Lea and Febiger, Philadelphia, 7th ed., 1918, 255.

4. Hewlett, A. W., Forchheimer's Therapeusis of Internal Diseases. New York, 1917, iii, 421. Diseases of the Lungs.

5. Macht, D. I., J. Pharm. and Exp. Therap., 1915, vii, 339. Action of the Opium Alkaloids, Individually and in Combination with Each Other, on the Respiration.

6. Higgins, H. L., and Means, J. H., J. Pharm. and Exp. Therap., 1915, vii,'1. The Effect of Certain Drugs on the Respiration and Gaseous Metabolism in Normal Human Subjects. 
7. Binger, C. A. L., and Davis, J. S., Jr., Proc. Soc. Exp. Biol. and Med., 1928, xxv, 607. A Body Plethysmograph for the Study of Respiratory Movements in Human Beings.

8. Van Slyke, D. D., and Neill, J. M., J. Biol. Chem., 1924, lxi, 523. The Determination of Gases in Blood and Other Solutions by Vacuum Extraction and Manometric Measurement.

9. Binger, C. A. L., and Davis, J. S., Jr., J. Clin. Invest., 1928, vi, 171. The Relation of Anoxemia to the Type of Breathing Seen in Pneumonia. 